${ }^{2}$ Academic Unit of Respiratory Medicine, University of Sheffield, Sheffield, UK: ${ }^{3}$ University of Edinburgh MRC Centre for inflammation research, Edinburgh, UK

Introduction and objectives The alveolar macrophage (AM) is a key player in orchestrating the inflammatory response to inhaled pathogens and environmental toxins. DEP causes the release of monocytes from the bone marrow, and during chronic inflammation, macrophages mature continuously from infiltrating monocytes. There are no studies investigating the chronic effects of DEP on monocytes and actions of DEP on neutrophils are unclear. We investigated how DEP modulate neutrophil and monocyte function and macrophage differentiation.

Methods Monocytes and neutrophils were obtained from healthy volunteers by density gradient centrifugation and negative magnetic selection. Monocyte-derived macrophages (MDMs) were generated in the presence or absence of DEP. Apoptosis and cell loss of MDMs were assessed by microscopy, propidium iodide positivity and alamar blue reduction assays. Killing assays of Streptococcus pneumoniae were performed. Cytokine generation in response to varied TLR agonists was assessed by ELISA and surface marker expression of receptors including TLRs, and CD14 was measured by FACS.

Results Monocytes and MDMs avidly phagocytosed DEP but neutrophils neither phagocytosed DEP, or markedly responded to DEP. However, chronic exposure to DEP in vitro had a detrimental effect on monocytes, with marked enhancement of apoptosis and cell loss. Despite this, the ability to kill an important respiratory pathogen $S$ pneumoniae was preserved. Cytokine generation to TLR agonists was reduced, and this phenotype was associated with a reduction in CD14 surface marker expression.

Conclusions DEP directly activate monocytes but not neutrophils, and chronic exposure may have detrimental effects on the host by enhancing loss of monocytes recruited to the airways and modulating aspects of the inflammatory response to pathogens.

\section{S148 REGULATION OF NEUTROPHIL APOPTOSIS BY PHOSPHOINOSITIDE 3-KINASES}

doi:10.1136/thx.2010.150946.49

${ }^{1} \mathrm{~J}$ K Juss, ${ }^{1} \mathrm{~A}$ M Condliffe, ${ }^{2} \mathrm{P}$ T Hawkins, , $\mathrm{L}$ Stephens, ${ }^{1} \mathrm{E}$ R Chilvers. ${ }^{1}$ Department of Medicine University of Cambridge, Cambridge, England; ${ }^{2}$ Babraham Institute, Babraham, England

Introduction Neutrophils are an essential component of the innate immune response. However, their microbicidal mechanisms (generation of reactive oxygen species and release of proteolytic enzymes) may contribute to tissue injury. Neutrophil-mediated tissue damage is a cardinal feature in the pathogenesis/progression of COPD, cystic fibrosis and certain types of asthma. Apoptosis is the key determinant of tissue neutrophil longevity and is critical to the resolution of granulocyte inflammation; pharmacological acceleration of neutrophil apoptosis can promote resolution of inflammation in animal models. The cytokine GM-CSF drives the aberrant neutrophil survival phenotype observed in patients with ARDS, and recent studies suggest the phosphoinositide 3-kinase (PI3K)/AKT pathway is pivotal in signalling GM-CSF-mediated neutrophil survival.

Hypothesis Given the emerging evidence that individual Class I PI3K isoforms $(\alpha, \beta, \delta$ and $\gamma$ ) exert non-redundant signalling roles and represent promising therapeutic targets in inflammation, we hypothesised a distinct contribution of individual Class I PI3K isoforms in mediating constitutive neutrophil apoptosis and the GM-CSF cytoprotective effect.

Methods We established techniques to isolate peripheral blood neutrophils from humans and from knockout/transgenic mice lacking functional PI3K isoforms to $95 \%$ purity, and used pan-PI3K inhibitor (LY294002), pan-Class I PI3K inhibitor (PI-103) and novel PI3K isoform-selective small molecule inhibitors (YM-024, TGX-
221, IC87114 and AS605240) to determine precise involvement of Class I PI3K isoforms $(\alpha, \beta, \delta$ and $\gamma$ ) in constitutive and GM-CSFdelayed neutrophil apoptosis. Apoptosis was quantified using morphology and annexin V-FITC/propidium iodide staining.

Results GM-CSF-mediated neutrophil survival was reversed by panPI3K inhibition but not by individual PI3K isoform inhibition. Combinatorial experiments suggest there is near-complete functional redundancy amongst Class I PI3Ks with regard to GM-CSFmediated inhibition of neutrophil apoptosis; additionally, neutrophils derived from double knockout $\mathrm{PI} 3 \mathrm{~K}-\delta / \gamma$ and PI3K- $\beta / \delta$ mice had normal constitutive apoptosis and GM-CSF mediated survival, but were sensitised to inhibition of the remaining isoforms.

Conclusions Thus Class I PI3Ks mediate GM-CSF survival of human and murine neutrophils but there is complete functional redundancy of the PI3K isoforms, necessitating multiple isoform inhibition to reverse GM-CSF-induced neutrophil survival. This finding informs our understanding of the mechanisms regulating neutrophil apoptosis and suggests neutrophil survival would be resilient to individual isoform-selective PI3K inhibitors.

\section{S149 CHARACTERISATION OF T CELL POPULATIONS IN THE LUNG}

doi:10.1136/thx.2010.150946.50

R Spruce, G Rankin, J Ward, S J Wilson, C Pickard, J A Warner. University of Southampton, Southampton, UK

Introduction Chronic obstructive pulmonary disease (COPD) is a chronic inflammatory condition characterised by increased numbers of neutrophils, macrophages and $\mathrm{CD}^{+} \mathrm{T}$ cells. However, recent evidence suggests that both $\mathrm{CD}^{+}$and $\mathrm{CD}^{+} \mathrm{T}$ cells can influence the pathogenesis of the disease. We aim to characterise the T cell subsets from both airway and parenchyma.

Method Macroscopically normal lung parenchyma and bronchial airway tissue was obtained from 10 patients (average age $=65.3 \pm 4.5$ years, $\quad F E V 1 / F V C=0.57 \pm 0.04$ ) undergoing lung resection for carcinoma and dissected into explants. Tissue explants were acetone fixed, embedded in GMA and sections immunostained for CD3, CD4, CD8 and for mast cells (AA1) as a positive control. Positive cells were counted and numbers corrected for tissue area. To examine $\mathrm{T}$ cell migration, explants from the same patients were incubated for $24 \mathrm{~h}$ at $37^{\circ} \mathrm{C}$. Cells that moved out of the explants into the supernatant were recovered, stained for T cell markers (CD3FITC, CD8-APC) and analysed via flow cytometry.

Results We found very few $\mathrm{T}$ cells in lung parenchyma $\left(\mathrm{CD} 3^{+}\right.$ cells $=1.1 \pm 0.3 / \mathrm{mm}^{2}$ ) though there were substantial numbers of mast cells $\left(65.6 \pm 13.9 / \mathrm{mm}^{2}\right)$. There were twice as many $\mathrm{CD}^{+}$cells compared to $\mathrm{CD}^{+}$cells. In contrast, the airway had more $\mathrm{T}$ cells present $\left(\mathrm{CD}^{+}=2.8 \pm 1.1 / \mathrm{mm}^{2}\right)$ and again $\mathrm{CD}^{+} \mathrm{T}$ cells predominated. We also used flow cytometry to characterise the $\mathrm{CD} 4: \mathrm{CD} 8$ ratio in Tcells that migrated out of the explants. Parenchymal supernatants contained substantial numbers of $\mathrm{T}$ cells with significantly more $\mathrm{CD}^{+}{ }^{+} \mathrm{T}$ cells than $\mathrm{CD}^{+}(\mathrm{CD} 4=28.5 \pm 4.1 \%, \mathrm{CD} 8=20.4 \pm 2.3 \%$, $\mathrm{p}<0.05)$. However in the airway, the ratio of CD4:CD8 cells was closer to $1: 1\left(\mathrm{CD}^{+}=26.5 \pm 7.4 \%, \mathrm{CD}^{+}=23.2 \pm 6.7 \%\right)$. Preliminary evidence suggests that the presence of mild/moderate airways obstruction does not affect the total number of T cells in either the parenchyma or the airway or the CD4:CD8 ratio in either compartment. There is also no evidence that the patient's smoking status affects T cell number or distribution.

Conclusion More $\mathrm{CD}^{+}$cells are seen in the bronchial tissue than in the parenchyma and the majority of these are $\mathrm{CD}^{+} . \mathrm{CD}^{+}$cells also migrate spontaneously out of the explants in both compartments and account for the majority of cells recovered in the supernatant. 